\title{
Thermal Properties of Silver Nanoparticle Sintering Bonding Paste for High-Power LED Packaging
}

\author{
Ping Zhang, ${ }^{1,2}$ Rongzhuan Wei, ${ }^{1}$ Jianhua Zeng, ${ }^{1}$ Miao Cai, ${ }^{1}$ Jing Xiao, ${ }^{1}$ and Daoguo Yang ${ }^{1}$ \\ ${ }^{1}$ Electromechanical Engineering College, Guilin University of Electronic Technology, No. 1 Jinji Road, Guilin, Guangxi 541004, China \\ ${ }^{2}$ Guangxi Experiment Center of Information Science, No. 1 Jinji Road, Guilin, Guangxi 541004, China \\ Correspondence should be addressed to Daoguo Yang; daoguo_yang@vip.163.com
}

Received 2 May 2016; Accepted 30 June 2016

Academic Editor: Yalin Dong

Copyright (c) 2016 Ping Zhang et al. This is an open access article distributed under the Creative Commons Attribution License, which permits unrestricted use, distribution, and reproduction in any medium, provided the original work is properly cited.

\begin{abstract}
This paper describes the preparation of low-temperature sintered nanosilver paste with inverse microemulsion method with Span80/Triton X-100 as the mixed-surfactant and analyzes the influence of different sintering parameters (temperature, pressure) on the shear properties of low-temperature sintering of nanosilver. Experimental results show that the shear strength of the lowtemperature sintering of nanosilver increases as the temperature and pressure increase. But there are many pores and relative fewer cracks on the sintering layer after low-temperature sintered. The test thermal resistance of low-temperature sintered nanosilver paste is $0.795 \mathrm{~K} / \mathrm{W}$ which is greater than SAC305 weld layer with a T3ster thermal analyzer. The adhesive performance and the heat dispersion of low-temperature sintered nanosilver paste need to be further researched and improved.
\end{abstract}

\section{Introduction}

In recent years, with the development of semiconductor technology, power electronic devices, and the lead-free materials, especially the emergence of wide-band gap semiconductor materials, packaging technology and interconnect materials face new challenges. For example, Silicon Carbide ( $\mathrm{SiC}$ ) and Gallium Nitride (GaN), with high breakdown electric field, high thermal conductivity, and excellent ability of radiation resistance, can operate normally in theory at high temperature up to $600^{\circ} \mathrm{C}$, but the traditional interconnect materials cannot meet the requirements of high temperature condition [1-3]. Due to the low cost, good wettability, and good mechanical properties, lead-tin solder has been widely used in high heat-flow density power devices [4]. However, the lead will harm more systems, including nerves, hematopoietic system, and internal secretion system, and result in cognitive ability decline when it enters the body. Solder alloy tends to have the disadvantage of low reliability at a high temperature. Besides, the chip is easy to fail due to the mismatch of the thermal expansion coefficient because of large thermal stress of solder alloy [5]. Conducting resin has lower thermal conductivity and is usually
$10 \sim 25 \mathrm{~W} / \mathrm{m} \cdot \mathrm{K}$ with lower glass inversion temperature $\mathrm{Tg}$. During the operation, the resin matrix of the conducting resin will gradually show fatigue failure and make the thermal resistance increase, which is extremely unfavorable to the heat dissipation and physical properties of the chip and severely limits the development in the high-power density devices $[6,7]$. Silver glass solder paste, as an alternative to lead-free interconnection between the chip and substrates, has high thermal conductivity and electric conductivity after sintering [8]. However, its sintering temperature is up to $600^{\circ} \mathrm{C}$ or even higher temperature. Besides, some processes also need pressure, which threatens the integrity and reliability of the chip and easily leads to device failure. Due to the fatal defects, it is difficult for the traditional interconnection materials to meet the development needs of high-power devices.

Compared with traditional interconnect materials, the functional phase of low-temperature sintered nanosilver paste is silver which has excellent electrical conductivity, thermal conductivity, and higher melting point (melting temperature of bulk silver is $960^{\circ} \mathrm{C}$ ) $[9,10]$; what is more, the low-temperature sintered nanosilver paste can be sintered under low temperature. All these superior performances make it widely concerned as new interconnection materials, 
especially used in high temperature, high-power, and high heat dissipation field and wide-band gap semiconductor equipment $[11,12]$.

This work describes the preparation of low-temperature sintered nanosilver paste with inverse microemulsion method with Span-80/Triton X-100 as the mixed-surfactant and analyzes the situation of inner of the shear layer after being sintered. Low-temperature sintered nanosilver paste is attempted to interconnect large-power Light-Emitting Diode (LED) and substrates. A T3ster thermal analyzer is used to test the thermal resistance of the sintering layer of low-temperature sintered nanosilver paste, and a contrast test is made with the common SAC305 solder paste.

\section{Materials and Methods}

Nanosilver paste is prepared with inverse microemulsion method with Span-80/Triton X-100 as the mixed-surfactant. First, mix Span 80, Triton X-100, n-hexyl alcohol, and normal heptane in proportion, stir the mixed solution at $30^{\circ} \mathrm{C}$ and low speed, drop $0.5 \mathrm{~mol} / \mathrm{mL}$ silver nitrate solution to prepare transparent yellow silver nitrate inverse microemulsion system, and then drop $0.1 \mathrm{~mol} / \mathrm{mL}$ hydrazine hydrate to prepare the inverse microemulsion containing silver nanoparticles. Finally, drop a certain amount of absolute ethyl alcohol to prepare low-temperature sintered nanosilver paste through cleaning and centrifugal deposition by centrifuge. Conduct thermogravimetric (TG) curve analysis and observe the distribution of nanosilver particles through scanning electron microscopy (SEM).

Nickel plating gold direct bonded copper (DBC) substrate is used as the substrate for shear strength test. In order to simplify the experiment, nickel plating gold DBC substrate of the same material is used as the analog chip. The sizes of the substrate and the analog chip are $5 \mathrm{~mm} \times 6 \mathrm{~mm}$ and $3 \mathrm{~mm}$ $\times 3 \mathrm{~mm}$, respectively. Low-temperature sintered nanosilver paste is coated by halftone printing with the printing thickness of $150 \mu \mathrm{m}$. Before coating, clean the substrate and the analog chip with anhydrous ethanol and distilled water to reduce the influence of impurities on the surfaces of substrate and analog chip and conduct three groups of shear test to the sintered samples, that is, $200^{\circ} \mathrm{C}$ sintering, $200^{\circ} \mathrm{C}$ low-pressure sintering, and $450^{\circ} \mathrm{C}$ sintering.

When conducting analysis to the thermal performance of low-temperature sintered nanosilver paste. Low-temperature sintered nanosilver paste is used for the connection between large-power LED chip and printed circuit board (PCB) substrate. The T3ster thermal analyzer is used to test the thermal resistance of the sintering layer of low-temperature sintered nanosilver paste, and a contrast test is made with the common SAC305 solder paste.

\section{Results and Discussion}

3.1. TG Curve and SEM Analysis of Low-Temperature Sintered Nanosilver Paste. Figure 1 shows TG curve analysis chart of low-temperature sintered nanosilver paste prepared through settlement after being cleaned with absolute ethyl alcohol,

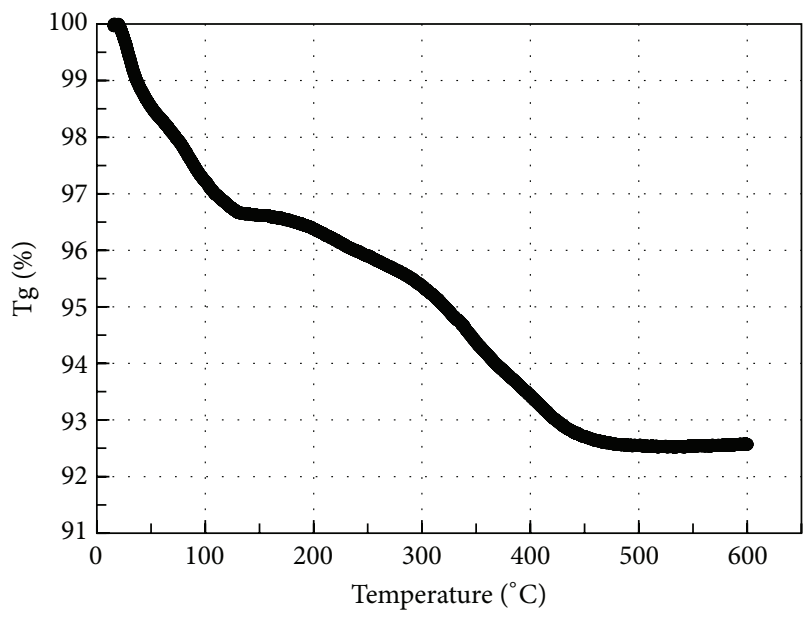

FIgURE 1: The TG curve of nanosilver paste.

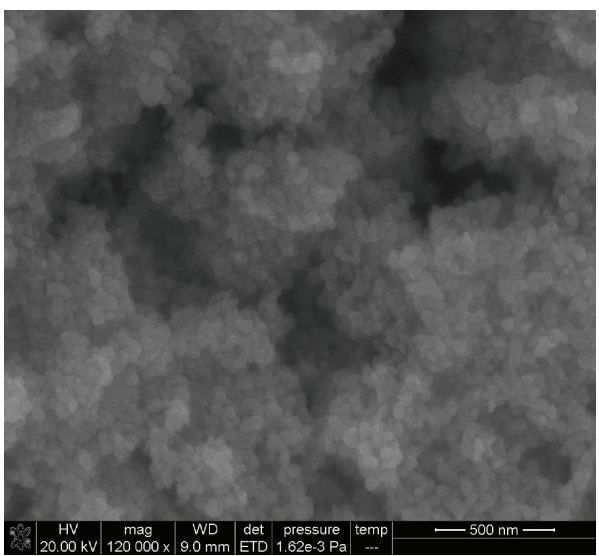

FIGURE 2: The SEM of nanosilver paste.

which analyzes the content of the components in lowtemperature sintered nanosilver paste and its decomposition temperature. We can learn from the figure that content of functional phase nanosilver particles in low-temperature sintered nanosilver paste is $92.57 \%$. TG curve can be divided into several stages below: ambient temperature $80^{\circ} \mathrm{C}$, the curve declining sharply due to the volatilization of absolute ethyl alcohol; $80^{\circ} \mathrm{C}-130^{\circ} \mathrm{C}$, the volatilization of normal heptane and n-hexyl alcohol; $130^{\circ} \mathrm{C}-450^{\circ} \mathrm{C}$, the curve declining slowly at the beginning and declining sharply when the temperature reaches $300^{\circ} \mathrm{C}$ mainly due to the decomposition and volatilization of Span 80 and Triton X-100 (the total content of Span 80 and Triton X-100 accounts for $4.1 \%$ of the total mass); and $450^{\circ} \mathrm{C}$ and above, the curve tending to be smooth because the dispersing agent and diluent used are nonionic organics. When the sintering temperature reaches $450^{\circ} \mathrm{C}$, the impurities can be negligible, and the agglomerate can be thought to be elemental silver and a small amount of silver oxide oxidized in the sintering process.

Figure 2 shows the particle size and morphology of as-prepared nanosilver particles after cleaning and vacuum drying observed with SEM. The drying temperature is about 


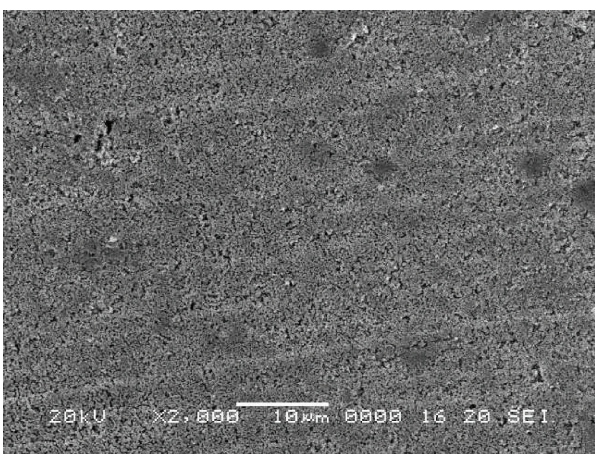

(a)

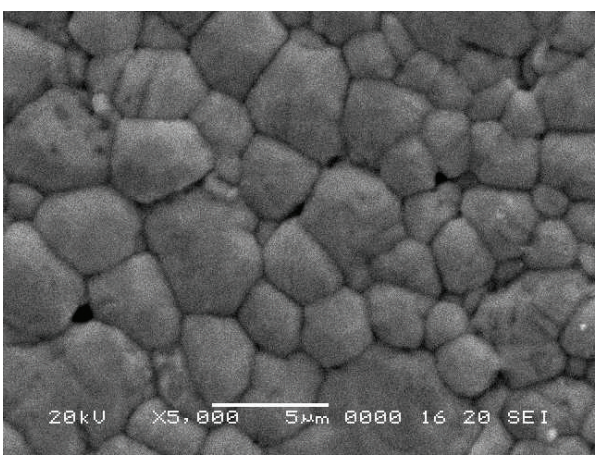

(c)

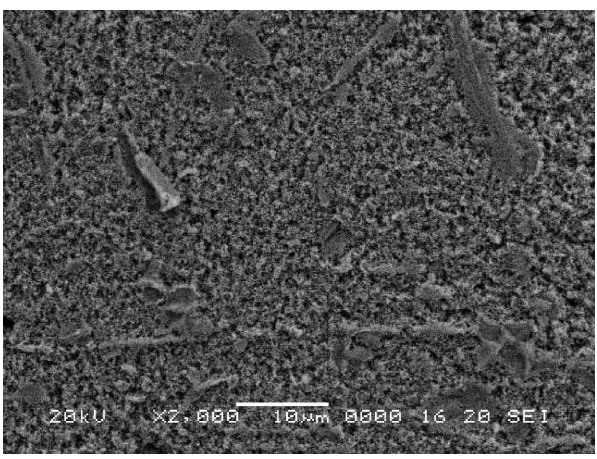

(e)

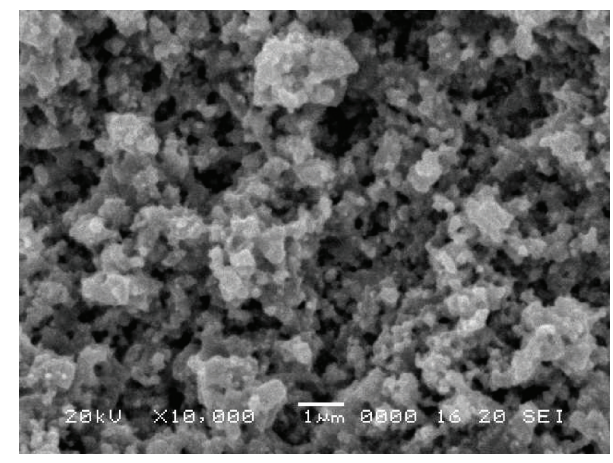

(b)

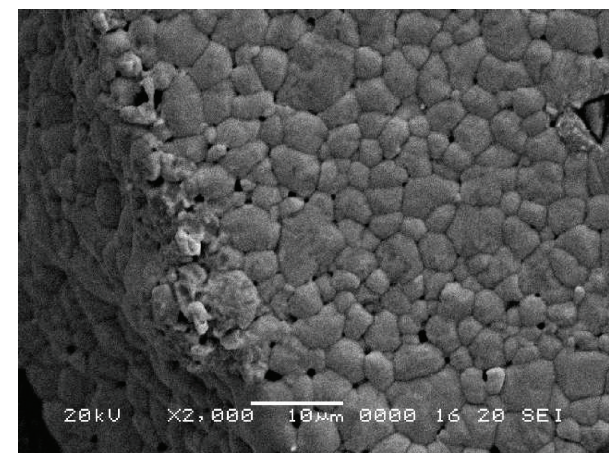

(d)

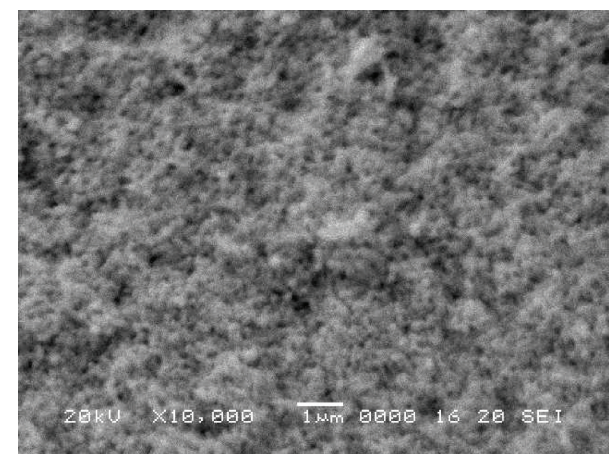

(f)

FIGURE 3: SEM images of interface microstructure of joints with different sintering temperatures and sintering pressure: $200^{\circ} \mathrm{C}$ sintering (a and b); $200^{\circ} \mathrm{C}$ low-pressure sintering ( $\mathrm{c}$ and $\mathrm{d}$ ); and $450^{\circ} \mathrm{C}$ sintering (e and f).

$40^{\circ} \mathrm{C}$. The nanosilver particles prepared by this method have uniform spherical particles, the distribution of nanosilver particle size is in the range of $30-60 \mathrm{~nm}$, and the average diameter of nanosilver particle size is about $40 \mathrm{~nm}$. The particle size of the nanosilver particles prepared in the method is larger may be because that the quantity of inverse microemulsion micelles is relatively small, and the nanosilver particles nucleate in the micelles and continue to grow due to limited quantity of inverse microemulsion micelles.

3.2. The Shear Performance of Low-Temperature Sintered Nanosilver Paste. When conducting shear test to $200^{\circ} \mathrm{C}$ sintered samples, the property of bonding between the lowtemperature sintered nanosilver paste and the substrate is reduced due to larger surface roughness of substrate and larger number of times of cleaning with absolute ethyl alcohol, making the maximum shear strength reach $12.25 \mathrm{MPa}$ by homemade shear model. Figure 3(a) shows the surface topography of the structure that low-temperature sintered nanosilver paste is agglutinated with the analog chip. There are many macropores and small black spots on the surface. It is mainly due to the presence of residual organic compounds in the paste. Figure 3(b) shows the structure and morphology of the inner shear off layer of the $200^{\circ} \mathrm{C}$ sintering layer. The morphology analysis of the broken crack is where the lowtemperature sintered nanosilver paste is sintered uniformly. However, there are many mesopores.

When the temperature is $200^{\circ} \mathrm{C}$ and the pressure is 1.11 MPa, the maximum shear strength of low-temperature sintered nanosilver paste can reach $18.7 \mathrm{MPa}$, which is larger 


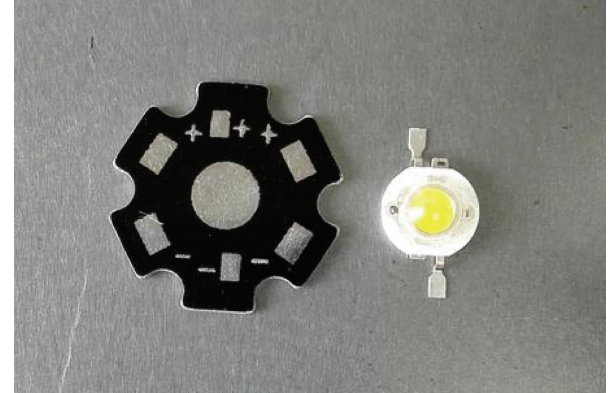

FIgURE 4: Large-power LED lamps and substrate.

than when there is no pressure. Figure 3(c) shows the morphology of the shear surface of low-temperature sintered nanosilver paste when the temperature is $200^{\circ} \mathrm{C}$ and the pressure is $1.11 \mathrm{MPa}$. The functional phase has a compact structure under the pressure. The sintered nanosilver particles are larger and uniform. Figure 3(d) shows the morphology of the shear fracture of low-temperature sintered nanosilver paste when the temperature is $200^{\circ} \mathrm{C}$ and the pressure is $1.11 \mathrm{MPa}$. The fracture structure has the same surface structure with the shear structure. The silver nanoparticles grow uniformly and are very compact.

The maximum shear strength of low-temperature sintered nanosilver paste can reach $27.36 \mathrm{MPa}$ when the temperature is $450^{\circ} \mathrm{C}$. Figure $3(\mathrm{e})$ shows the fractured surface topography of the sintering shearing interface at the temperature of $450^{\circ} \mathrm{C}$. There are layers of thin sheets. The morphology is different from that when black stripes and spots appear during sintering at the temperature of $200^{\circ} \mathrm{C}$. These sheets may be mainly the shedding layer of the surface of the analog chip, which ultimately leads to the decrease of shear strength. Figure 3(f) shows the sintering fracture structure of low-temperature sintered nanosilver paste at the temperature of $450^{\circ} \mathrm{C}$. The inner fracture structure of the sintered layer has few cracks and mesopores. Functional phase of lowtemperature sintered nanosilver particles sintered at the low temperature grow and connect tightly.

\subsection{The Thermal Performance of Low-Temperature Sintered} Nanosilver Paste. When conducting analysis to the thermal performance of low-temperature sintered nanosilver paste, the rated power of high-power LED is $3 \mathrm{~W}$, and the input current is $650 \mathrm{~mA}$, as shown in Figure 4. Number I LED lamps are represented as low-temperature sintered nanosilver paste interconnecting large-power LED lamps and the substrate, and number II LED lamps are represented as SAC305 interconnecting large-power LED lamps and the substrate. The sintering temperature for interconnection of number I LED lamps is $200^{\circ} \mathrm{C}$, and the welding temperature for interconnection of number II LED lamps is $210^{\circ} \mathrm{C}$. T3Ster equipment is used to analyze the coefficient of $K$ and the thermal resistance of the two types of LED lamp samples. The results show that the optical power of SAC305 at the working current of $650 \mathrm{~mA}$ is $0.3932 \mathrm{~W}$, and the coefficient of $K$ is $-2.152 \mathrm{mV} /{ }^{\circ} \mathrm{C}$, the optical power of low-temperature

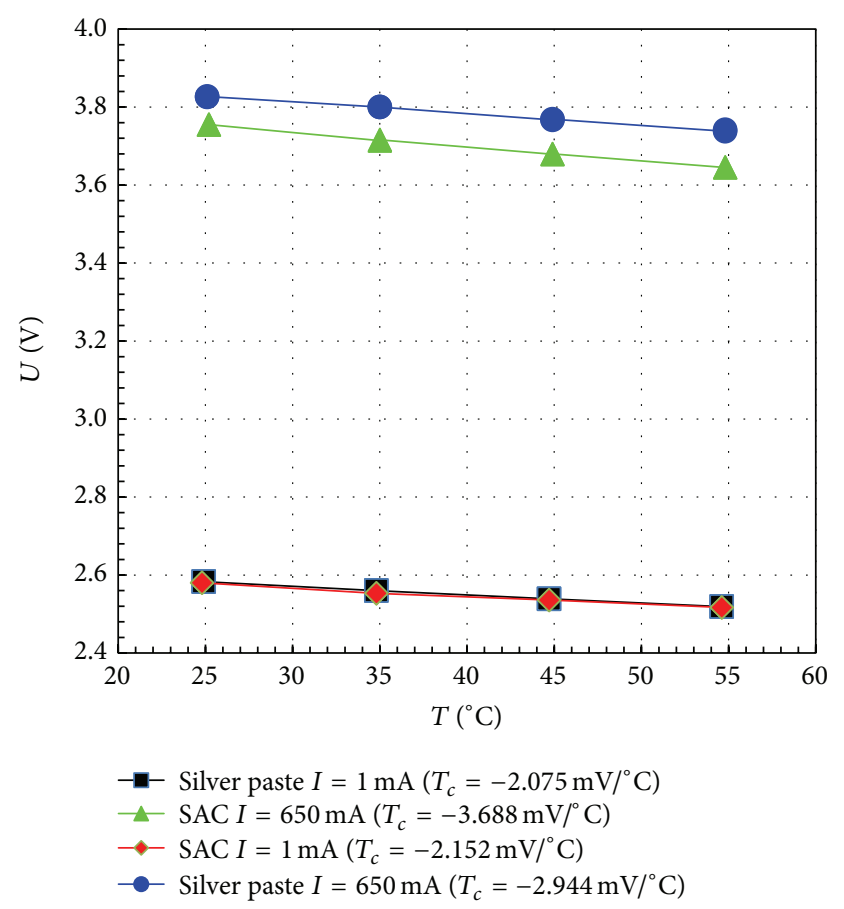

FIgURE 5: The coefficient $K$ curve of nanosilver paste and SAC305.

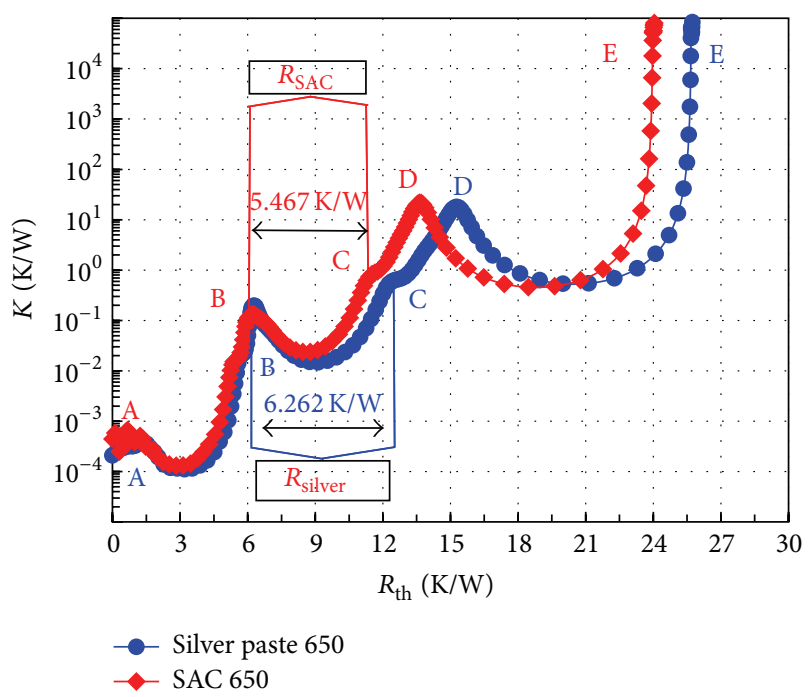

FIGURE 6: The comparison diagram of differential structure curves of number I LED lamps and number II LED lamps.

sintered nanosilver paste at the working current of $650 \mathrm{~mA}$ is $0.3932 \mathrm{~W}$, and the coefficient of $K$ is $-2.075 \mathrm{mV} /{ }^{\circ} \mathrm{C}$, the coefficient $K$ curve as shown in Figure 5 . We can see from the figures that coefficient $K$ of the two samples changes not so much, also it shows that the LED chip has stable quality.

Figure 6 is the comparison diagram of differential structure functions of number I LED lamps and number II LED lamps. As shown in the figure, in both of these functions the local peaks and valleys indicate reaching new materials or changed surface areas in the heat-flow path. Peaks correspond 
to regions of high thermal conductivity and valleys show region of low thermal conductivity, we can see that the thermal resistance of the low-temperature sintered nanosilver paste is $6.262 \mathrm{~K} / \mathrm{W}$, and the thermal resistance of SAC305 is $5.467 \mathrm{~K} / \mathrm{W}$. The thermal resistance of low-temperature sintered nanosilver paste is $0.795 \mathrm{~K} / \mathrm{W}$ larger than that of SAC305. The junction temperature of the two types of LED lamps is obtained by processing the temperature change curves with software, which are $79.38^{\circ} \mathrm{C}$ and $75.34^{\circ} \mathrm{C}$ for number I and number II LED lamps. Because the thermal resistance of the low-temperature sintered nanosilver paste after sintering is greater than that of SAC305, causing that the junction temperature of number I LED lamps is larger than that of number II LED lamps. It also shows that the thermal performance after sintering of low-temperature sintered nanosilver paste used in this kind of LED lamp is worse than that used in SAC305. This is because the larger surface roughness of large-power LED heat dissipation block and PCB substrates and poor adhesive performance cause more pores on the sintering layer and larger interface resistance [13]; on the one hand, high-power LED heat dissipation block is made of aluminum which is easy to be oxidized, and the diffusion of silver atoms is worse on the aluminum surface than on the gold surface [14]. And SAC305 has good wettability and adhesive performance on aluminum block and PCB substrates, greatly reducing the thermal resistance of the interface.

\section{Conclusions}

(1) Low-temperature sintered nanosilver paste is prepared with inverse microemulsion method with Span-80/Triton X-100 as the mixed-surfactant. The shear strength of the low-temperature sintering of nanosilver under low pressure is higher, the pore is smaller, and the particles after sintering are larger and the arrangement is compact. However, there are more cracks.

(2) The surface of the heat dissipation block and PCB is rougher, and the surface material is nonsilver material, which affects the diffusion of silver atoms during the sintering of low-temperature sintered nanosilver paste and results in poor adhesive performance. Finally, the test thermal resistance of low-temperature sintered nanosilver paste is $0.795 \mathrm{~K} / \mathrm{W}$ higher than that of SAC305 in the application.

(3) Adding functional phase materials, such as copper and graphite in low-temperature sintered nanosilver paste, may effectively improve the adhesive performance and the heat dispersion, which needs further research and improvement.

\section{Competing Interests}

The authors declare that there is no conflict of interests regarding the publication of this paper.

\section{Acknowledgments}

The authors acknowledge the financial support provided by supported by National Natural Science Foundation of China (Project no. 51506033), Guangxi University of Science and Technology Research Projects (Grant no. KY2015ZD045), Innovation Project of GUET Graduate Education (Grant no. 2016YJCX18), and Guangxi's Key Laboratory Foundation of Manufacturing Systems and Advanced Manufacturing Technology (Grant no. 15-140-30-005Z).

\section{References}

[1] J. Hornberger, A. B. Lostetter, K. J. Olejniczak, T. McNutt, S. M. Lal, and A. Mantooth, "Silicon-carbide (SiC) semiconductor power electronics for extreme high-temperature environments," in Proceedings of the IEEE Aerospace Conference Proceedings, pp. 2538-2555, Big Sky, Mont, USA, March 2004.

[2] T. Nomura, M. Masuda, N. Ikeda, and S. Yoshida, "Switching characteristics of GaN HFETs in a half bridge package for high temperature applications," IEEE Transactions on Power Electronics, vol. 23, no. 2, pp. 692-697, 2008.

[3] R. Khazaka, B. Thollin, L. Mendizabal, D. Henry, R. Khazaka, and R. Hanna, "Characterization of nanosilver dry films for high-temperature applications," IEEE Transactions on Device and Materials Reliability, vol. 15, no. 2, pp. 149-155, 2015.

[4] L. R. Garcia, W. R. Osório, L. C. Peixoto, and A. Garcia, "Mechanical properties of $\mathrm{Sn}$-Zn lead-free solder alloys based on the microstructure array," Materials Characterization, vol. 61, no. 2, pp. 212-220, 2010.

[5] C.-J. Chen, C.-M. Chen, R.-H. Horng, D.-S. Wuu, and J.S. Hong, "Thermal management and interfacial properties in high-power GaN-based light-emitting diodes employing diamond-added Sn-3 wt.\%Ag- 0.5 wt.\% Cu solder as a die-attach material," Journal of Electronic Materials, vol. 39, no. 12, pp. 2618-2626, 2010.

[6] P. Hu, W. O'Neil, and Q. Hu, "Synthesis of $10 \mathrm{~nm}$ Ag nanoparticle polymer composite pastes for low temperature production of high conductivity films," Applied Surface Science, vol. 257, no. 3, pp. 680-685, 2010.

[7] W. Tao, S. Chen, P. Berggren, and J. Liu, "Reliability study for high temperature stable conductive adhesives," in Proceedings of the International Symposium on Advanced Packaging Materials: Microtech (APM '10), pp. 74-77, IEEE, Cambridge, UK, March 2010.

[8] R. Kisiel, Z. Szczepański, M. Sochacki et al., "Thermal properties of SiC-ceramics substrate interface made by silver glass composition," in Proceedings of the 34th International Spring Seminar on Electronics Technology (ISSE '11), pp. 98-102, IEEE, Tratanska Lomnica, Slovakia, May 2011.

[9] W. Guo, Z. Zeng, X. Zhang, P. Peng, and S. Tang, "Lowtemperature sintering bonding using silver nanoparticle paste for electronics packaging," Journal of Nanomaterials, vol. 2015, Article ID 897142, 7 pages, 2015.

[10] P. Peng, A. Hu, A. P. Gerlich, G. Zou, L. Liu, and Y. N. Zhou, "Joining of silver nanomaterials at low temperatures: processes, properties, and applications," ACS Applied Materials \& Interfaces, vol. 7, no. 23, pp. 12597-12618, 2015.

[11] P. Ning, T. G. Lei, F. Wang, G.-Q. Lu, K. D. T. Ngo, and K. Rajashekara, "A novel high-temperature planar package for SiC multichip phase-leg power module," IEEE Transactions on Power Electronics, vol. 25, no. 8, pp. 2059-2067, 2010. 
[12] R. Khazaka, L. Mendizabal, and D. Henry, "Review on joint shear strength of nano-silver paste and its long-term high temperature reliability," Journal of Electronic Materials, vol. 43, no. 7, pp. 2459-2466, 2014.

[13] L. Jiang, T. G. Lei, K. D. T. Ngo, G.-Q. Lu, and S. Luo, "Evaluation of thermal cycling reliability of sintered nanosilver versus soldered joints by curvature measurement," IEEE Transactions on Components, Packaging and Manufacturing Technology, vol. 4, no. 5, pp. 751-761, 2014.

[14] E. Ide, S. Angata, A. Hirose, and K. F. Kobayashi, "Bonding of various metals using Ag metallo-organic nanoparticles-a novel bonding process using Ag metallo-organic nanoparticles," in Materials Science Forum, pp. 383-388, Trans Tech Publications, 2006. 

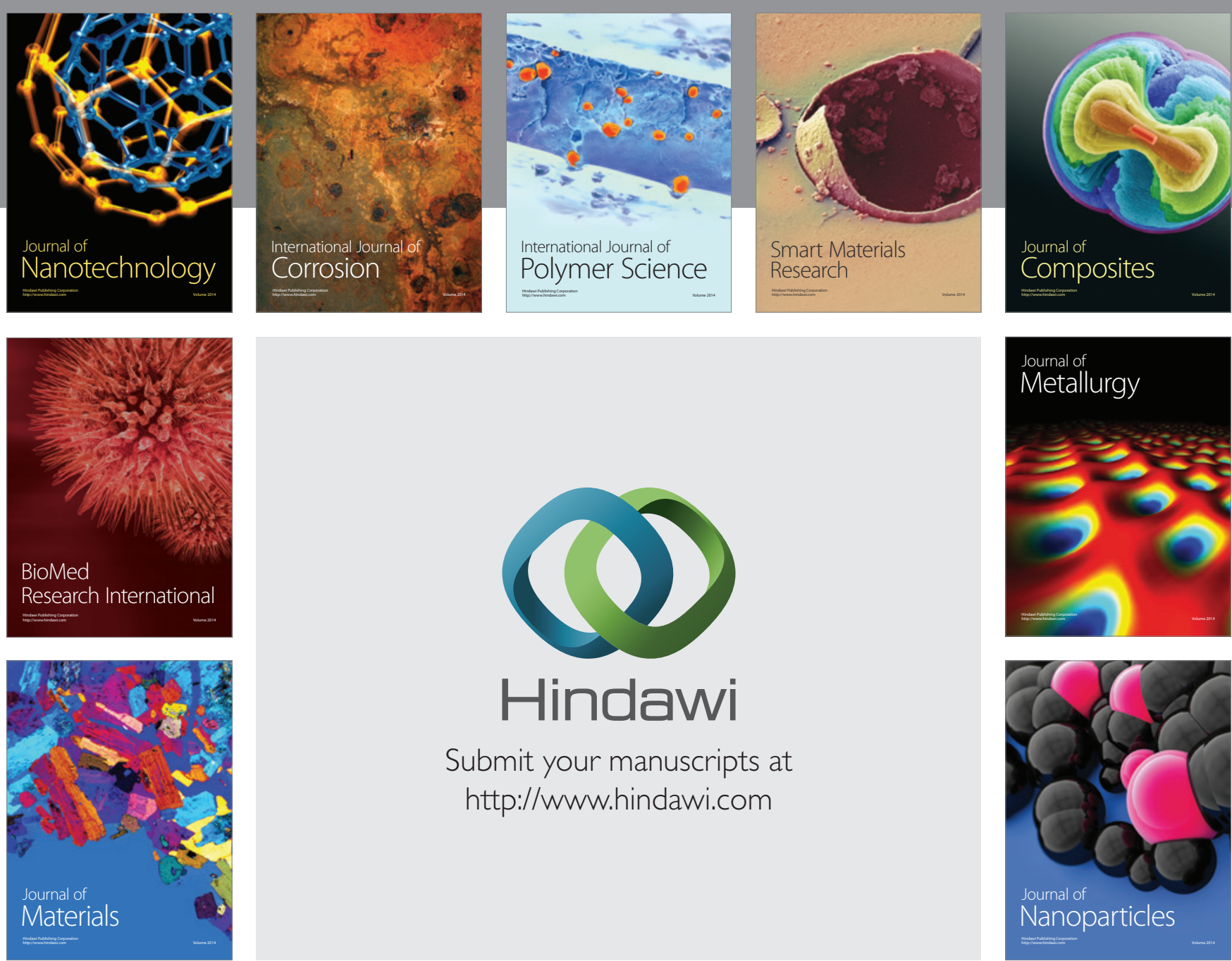

\section{Hindawi}

Submit your manuscripts at

http://www.hindawi.com

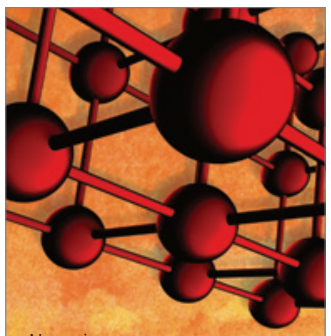

Materials Science and Engineering
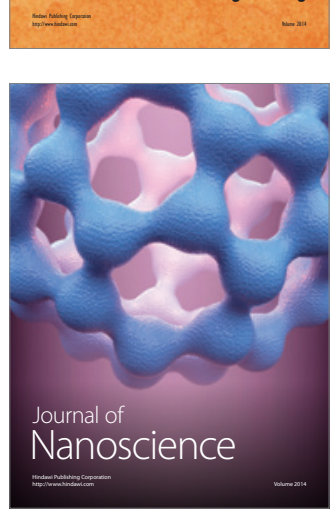
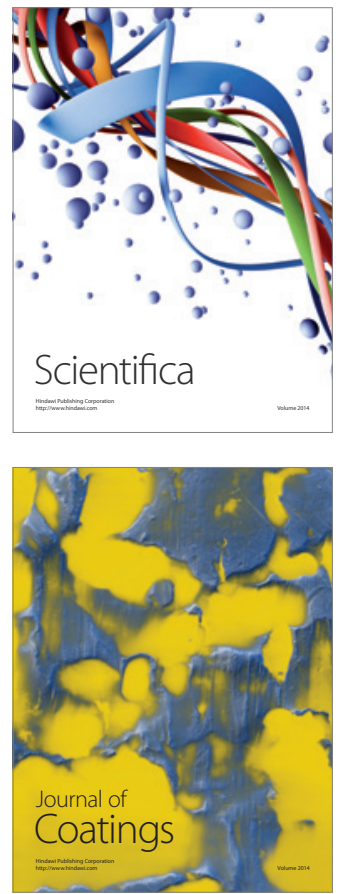
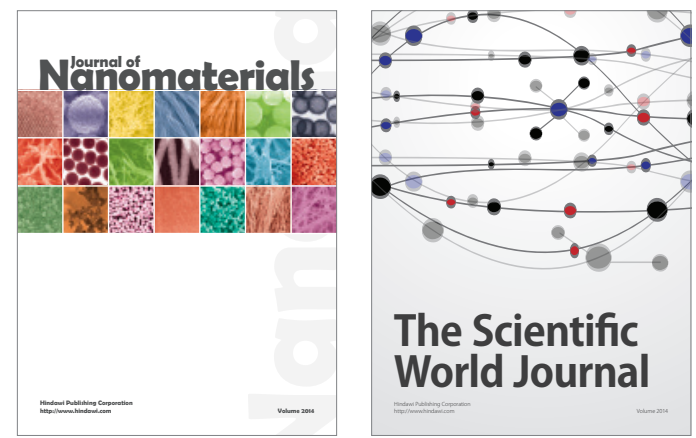

The Scientific World Journal
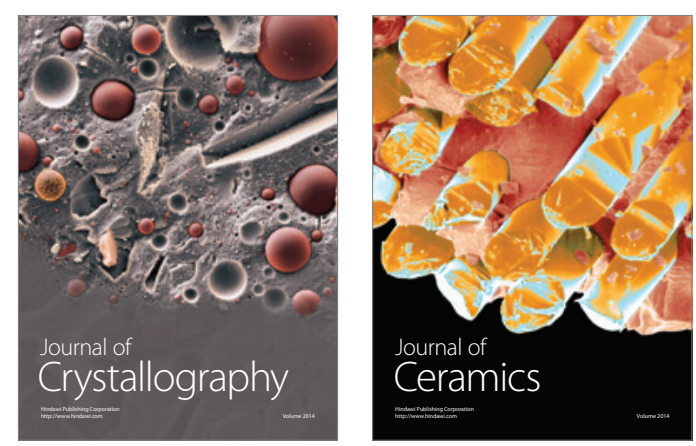
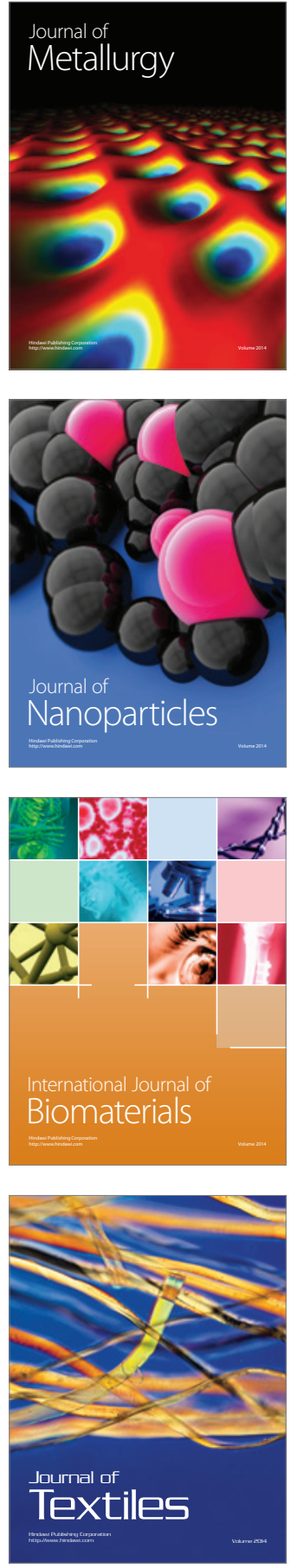